\title{
IN CYLINDER COLD FLOW CFD SIMULATION OF IC ENGINE USING HYBRID APPROACH
}

\author{
Pathak Yogesh $\mathbf{R}^{1}$, Deore Kailas $\mathbf{D}^{2}$, Patil Vijayendra $\mathbf{M}^{3}$ \\ ${ }^{1}$ Asst. Professor, R.C.P.I.T. Shirpur, Maharashtra. India \\ ${ }^{2}$ Asst. Professor, R.C.P.I.T. Shirpur, Maharashtra. India \\ ${ }^{3} P G$ Student, Technocrats Institute of Technology, Bhopal, M.P. India
}

\begin{abstract}
The design and manufacture of Internal Combustion (IC) Engines is under significant pressure for improvement. The next generation of engines needs to be compact, light, powerful, and flexible, yet produce less pollution and use less fuel. Innovative engine designs will be needed to meet these competing requirements. The time scales of the intake air flow, fuel injection, liquid vaporization, turbulent mixing, species transport, chemistry, and pollutant formation all overlap, and need to be considered simultaneously. Computational Fluid Dynamics (CFD) has emerged as a useful tool in understanding the fluid dynamics of IC Engines for design purposes. . Insight provided by CFD analysis helps guide the geometry design of parts, such as ports, valves, and pistons; as well as engine parameters like valve timing and fuel injection. Using CFD results, the flow phenomena can be visualized on $3 D$ geometry and analyzed numerically, providing tremendous insight into the complex interactions that occur inside the engine. This allows you to compare different designs and quantify the trade-offs such as soot vs NOx, swirl vs tumble and impact on turbulence production, combustion efficiency vs pollutant formation, which helps determine optimal designs. In this paper the In cylinder cold flow CFD simulation of four stroke petrol engine using hybrid approach of ANSYS fluent. The simulation is carried out using parameter and journal files which is a symmetry geometry. Dynamic motion was visualize and velocity magnitude is plotted for crank angle starting from 0 to 720 . The engine is simulated for half cylinder cycle. The In cylinder data file is write displaying swirl and tumble for zones of fluid-ch and fluid-piston-layer. The text file is written in working directory containing swirl, $x$-tumble, $y$-tumble and moment of inertia as a function of CA.
\end{abstract}

Keywords: CFD, In-Cylinder analysis, cold flow Simulation, IC Engine Analysis.

\section{INTRODUCTION}

Now a day's engineers are looking for new methodologies for improving the functionality of the product using various approaches which will reduce cost of research and give the desired results. CFD is such a technique which has emerged from last few decades as a important part in research of various systems finding its applications in near about every engineering disciplines. This paper describes the use of CFD method for simulation and analysis of internal combustion engine cold flow and combustion, which is tedious and complicated by experimentation. Using CFD methods we can get optimum results with no cost of experimentation and in less time. ANSYS/FLUENT is the CAE package which can be used for such simulations for accurate results and due to better meshing capabilities for sophisticated problems.

The design and manufacture of Internal Combustion (IC) Engines is under significant pressure for improvement. The next generation of engines needs to be compact, light, powerful, and flexible, yet produce less pollution and use less fuel. Innovative engine designs will be needed to meet these competing requirements. The ability to accurately and rapidly analyze the performance of multiple engine designs is critical. The performance of an IC Engine depends upon complex interactions between mechanical, fluid, chemical, and electronic systems. However, the central challenge in design is the complex fluid dynamics of turbulent reacting flows with moving parts through the intake/exhaust manifolds, valves, cylinder, and piston. The time scales of the intake air flow, fuel injection, liquid vaporization, turbulent mixing, species transport, chemistry, and pollutant formation all overlap, and need to be considered simultaneously. Computational Fluid Dynamics (CFD) has emerged as a useful tool in understanding the fluid dynamics of IC Engines for design purposes. This is because, unlike analytical, experimental, or lower dimensional computational methods, multidimensional CFD modeling allows designers to simulate and visualize the complex fluid dynamics by solving the governing physical laws for mass, momentum, and energy transport on a 3D geometry, with sub-models for critical phenomena like turbulence and fuel chemistry. Insight provided by CFD analysis helps guide the geometry design of parts, such as ports, valves, and pistons; as well as engine parameters like valve timing and fuel injection. Using CFD results, the flow phenomena can be visualized on $3 \mathrm{D}$ geometry and analyzed numerically, providing tremendous insight into the complex interactions that occur inside the engine. This allows you to compare different designs and quantify the trade-offs such as soot vs NOx, swirl vs tumble and impact on turbulence production, combustion efficiency vs pollutant formation, which helps determine optimal designs. Hence CFD analysis is used extensively as part of the design process in automotive engineering, power generation, and transportation. With the 
rise of modern and inexpensive computing power and 3D CAD systems, it has become much easier for analysts to perform CFD analysis. In increasing order of complexity, the CFD analyses performed can be classified into Port Flow Analysis: Quantification of flow rate, swirl and tumble, with static engine geometry at different locations during the engine cycle.

Cold Flow Analysis: Engine cycle with moving geometry, air flow, and no fuel injection or reactions.

In-Cylinder Combustion Simulation: Power and exhaust strokes with fuel injection, ignition, reactions, and pollutant prediction on moving geometry.

Full Cycle Simulation: Simulation of the entire engine cycle with air flow, fuel injection, combustion, and reactions.

\section{COLD FLOW IN-CYLINDER ANALYSIS}

Cold flow analysis involves modeling the airflow and possibly the fuel injection in the transient engine cycle without reactions. The goal is to capture the mixture formation process by accurately accounting for the interaction of moving geometry with the fluid dynamics of the induction process. The changing characteristics of the air flow jet that tumbles into the cylinder with swirl via intake valves and the exhaust jet through the exhaust valves as they open and close can be determined, along with the turbulence production from swirl and tumble due to compression and squish. Two approaches are employed in ANSYS FLUENT to solve in-cylinder (IC) problems, namely, hybrid approach and layering approach. The layering approach is used for engines with vertical valves like most diesel engines, while the hybrid approach is typically used for engines with canted valves like most spark ignited (SI) engines. There are three stages for IC simulation. Decompose the geometry into different zones and mesh them properly. By breaking up the model into different zones, it is possible to apply different mesh motion strategies to different regions in a single simulation. Set up the engine case in ANSYS FLUENT with the help of a setup journal. Perform a transient IC simulation. the simulation of the engine starts by import an engine geometry. The imported geometry is divided into smaller volumes before meshing. This enables each volume to be meshed separately. Decomposition partitions a volume into sub-volumes and then the sub-volumes are meshed individually. Each volume will be meshed into hex or tet elements, depending upon the approach. There are certain mesh topology requirements for valves and pistons. You should have pistons at TDC (top dead center) and valves at closed position before the geometry is decomposed. With the piston at TDC, the volume is the smallest. In general it is more difficult to satisfy the mesh topology requirement at TDC, but this provides the advantage that the mesh will behave properly when the piston moves away from TDC. For the same reason, the valves should be at the closed position for the geometry. However, the simulation requires a minimum valve lift between the valve and valve seat so that layered cells can be placed at the region of minimum valve lift. This ensures that the gap between the valve and valve seat will not disappear. A non-conformal interface is used to completely shut the valve. Even though in theory an arbitrarily small minimum valve lift can be used, in reality a value of $0.05 \mathrm{~mm}$ to $0.5 \mathrm{~mm}$ has been successfully used to run simulations using ANSYS FLUENT.

The CAD model in native format can be imported to ANSYS/Fluent. After importing the CAD file the engine geometry is meshed into different volumes separate mesh is used for separate volumes in the engine table 1 shows the different fluid zones and mesh requirements.

Table 1 Mesh Requirements for fluid zones

\begin{tabular}{|l|l|l|}
\hline Sr.No & Fluid Zone Name & Mesh Requirement \\
\hline 1 & fluid-ch & tet mesh \\
\hline 2 & fluid-rootname-ib & layered mesh \\
\hline 3 & fluid-rootname-port & any mesh \\
\hline 4 & fluid-rootname-vlayer & layered mesh \\
\hline 5 & fluid-piston-layer & layered mesh \\
\hline
\end{tabular}

Meshing should be properly defined in case because the parts of the engines such as the piston and valves are provided with dynamic mesh. As these parts are in motion during analysis improper mesh for such fluid zones will cause failure in analysis.

\subsection{Case Description}

The engine in this paper is four stroke single cylinder petrol engine with canted valves one inlet and one exhaust valve. It is a in-cylinder engine having piston and cylinder in in line. The model is prepared with cad software meshing is carried out. The case file is imported in ANSYS/Fluent (3D). This paper considers a 3D symmetric geometry of a IC engine cylinder configuration. Case setup is performed using a scheme file that automatically sets up necessary motions for valves and pistons along with solution parameters found to be best suit for the in-cylinder simulation. The journal file automatically sets up the necessary motions for valves and piston, along with solution parameter which suit the IC simulation best. Journal file allows you to set up an IC simulation with all the best practices built-in, without learning the dynamic mesh capability. This makes use of a In-Cylinder Output Controls to calculate swirl and tumble ratio. The case has a symmetric plane. Use of symmetry helps reduce cell count by a factor of two and thus greatly reduce the run time. Layering is used for lower combustion chamber and the valve seat, to reduce cell count and to properly resolve the flow. Tetrahedral mesh is used in the upper combustion chamber and ports to facilitate the setup. The mesh must have the correct decomposition and names before using the journal to automatically set up the case. 


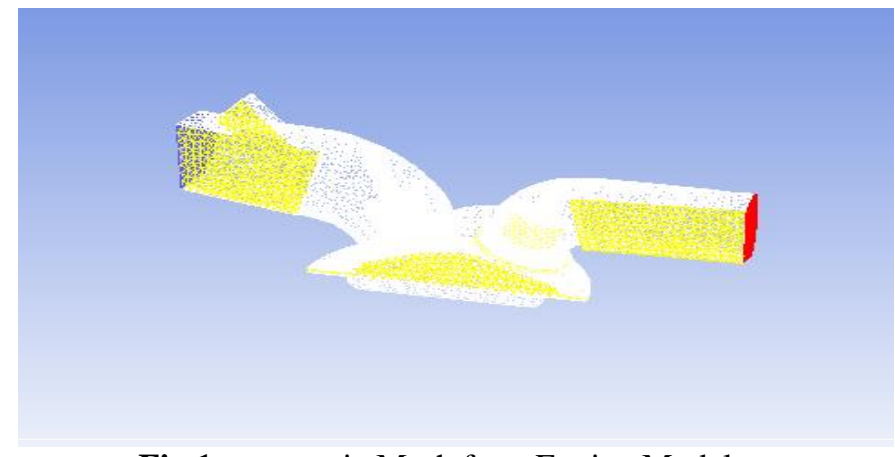

Fig 1 symmetric Mesh for a Engine Model

Here you have to provide the path to the profile file. This file essentially contains the valve lift values at different crank angles. It can also include the piston motion profile. You can use this profile file to animate the movement of the valves. It is also used for simulating the valve motion. The format of the profile file is fairly simple. The file can contain an arbitrary number of profiles. Profile names must have all lowercase letters. Here profile-name is the name given to the type of valve. For example, it can be invalve1 or exvalve2. The types of profile are point and transient. The mandatory field-names are angle and lift. Parentheses are used to delimit profiles and the fields within the profiles. Any combination of tabs, spaces, and newlines can be used to separate elements. The next step is to define the In cylinder mesh motion setup using the parameter file. The file contains all the engine parameters and parameters required for valves motion and piston motion setup. The details of parameter file are shown in table 2.

Table 2 Details of Parameter file

\begin{tabular}{|l|l|l|}
\hline Sr. & Parameter & Value \\
\hline 1 & Engine Speed $(\mathrm{rpm})$ & 1200 \\
\hline 2 & Crank angle step size $(\mathrm{m})$ & 0.25 \\
\hline 3 & Piston Stroke $(\mathrm{m})$ & 0.085 \\
\hline 4 & Connecting rod length $(\mathrm{m})$ & 0.147 \\
\hline 5 & Min Valve lift $(\mathrm{m})$ & 0.0005 \\
\hline
\end{tabular}

The journal will automatically set up mesh motion for the intake valve, exhaust valve, and piston. The scheme file will automatically select Standard k-e as the turbulence model. The valve lift profile can be examine by the graph shown in fig 2. In convention with ANSYS FLUENT, the 0 crank angle (CA) is at top dead center (TDC) after compression. So, at $0 \mathrm{CA}$ both intake and exhaust valves are closed as shown in Fig 2. The graph shows crank angle vs time plot. The crank angle ranges from 0 to 720 for inlet and exhaust valve. Zone motion preview will translate moving zones, without solving any mesh or flow equations. This helps to quickly check valve profile and axis definition, that you put into the IC Setup dialog box. The dynamic mesh motion preview is then performed to display the movement of piston and inlet, exhaust valves starting from 0 crank angle. i.e from TDC location of piston A full 720 degree of mesh motion may not be necessary. But it is necessary to move the mesh to the simulation starting CA that is normally a few degrees before intake valve open at TDC. In this case,
CA 344 is the starting point of the simulation because the intake valve opens at CA 349 ..

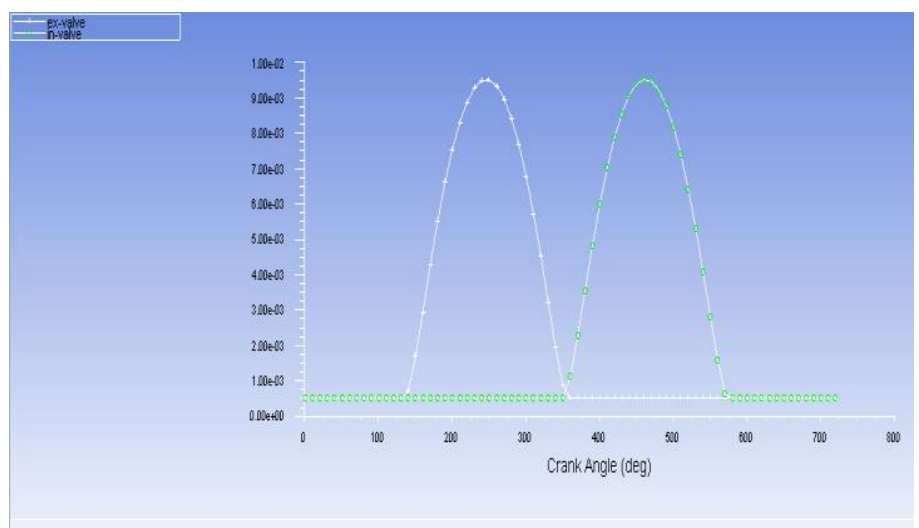

Fig 2 Inlet and exhaust valve lift profile

the next step is to preview mesh motion of piston and valves for required crank angles. Boundary conditions are then specified at inlet and outlet. Inlet boundary condition is specified with Intensity and Hydraulic Diameter from turbulence Intensity with 5\% Turbulent Intensity and $0.03 \mathrm{~m}$ for Hydraulic diameter. The total temperature is $300 \mathrm{~K}$. for outlet boundary condition Backflow Turbulent Intensity is set to $8 \%$ Backflow Hydraulic Diameter is $0.03 \mathrm{~m}$ and total temperature is $300 \mathrm{~K}$. in next step the In cylinder output controls are defined. Specifying various quantities needed for the calculation of swirl and tumble along with the frequency of writing the output, to the chosen _le. Swirl is used to describe circulation about the cylinder axis. Tumble flow circulates around an axis perpendicular to the cylinder axis, orthogonal to swirl flow. The In cylinder data file is write displaying swirl and tumble for zones of fluid-ch and fluid-piston-layer. The text file is written in working directory containing swirl, x-tumble, y-tumble and moment of inertia as a function of CA. finally, solution is initialize and iso-surfaces are created for better visualization of the results and velocity magnitude contour is selected for results. 1440 time step simulation is run for a half cycle of the cylinder for full cycle simulation 2880 time step is selected. Here, in this paper half cycle simulation is carried out the results of velocity magnitude for various crank angles are shown in fig $3,4 \& 5$.

\section{RESULTS}

The various parameters such Tumble, Swirl and Moment of Inertia are compared in graphs shown in fig 5,6,7,8,9,10 \& 11 .

where,

$\mathrm{CA}=$ Crank Angle .

$\mathrm{L}=$ Angular momentum vector of fluid mass contained in selected cell zones with respect to swirl center..

$\mathrm{sa}=$ Swirl axis.

$\mathrm{tx}=$ Tumble $\mathrm{x}$-axis.

ty $=$ Tumble $\mathrm{y}$-axis.

$\mathrm{I}_{\mathrm{sa}}=$ Moment of Inertia of the fluid mass about swirl axis.

$\mathrm{I}_{\mathrm{tx}}=$ Moment of Inertia of the fluid mass about tumble $\mathrm{x}$ axis. 
$\mathrm{I}_{\mathrm{ty}}=$ Moment of Inertia of the fluid mass about tumble $\mathrm{y}$ axis.
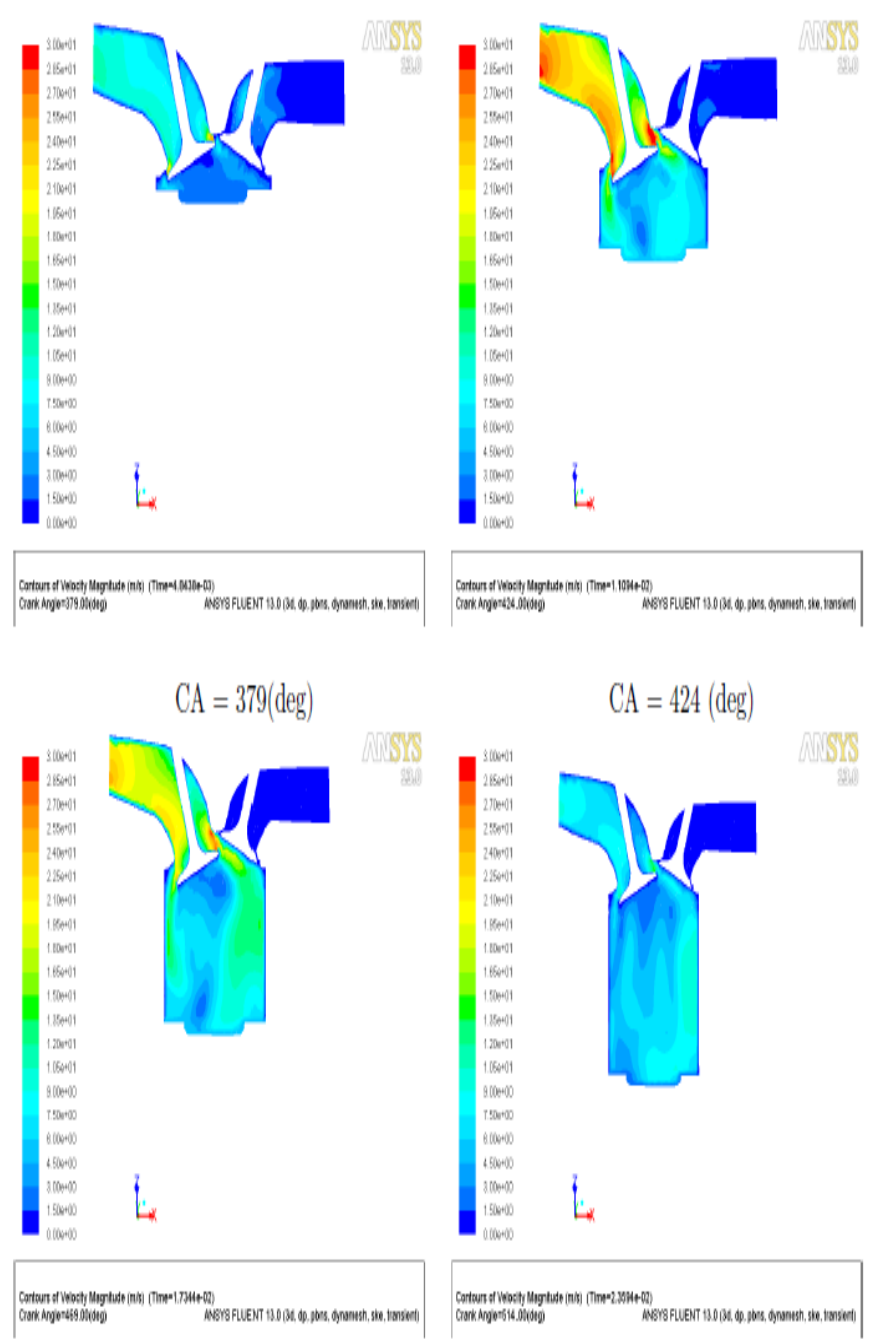

Fig 3 Velocity magnitude for CA 379,424,469 and 514
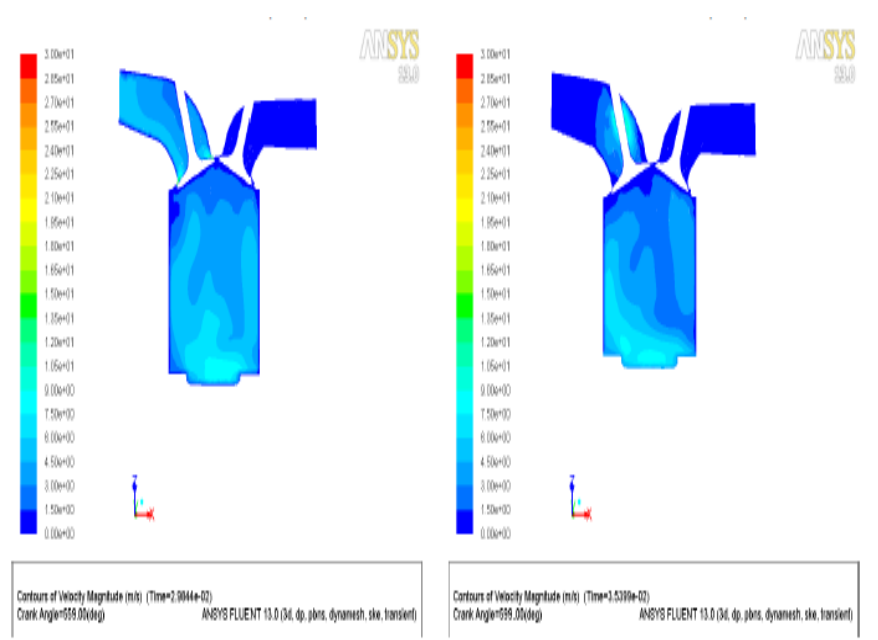

$$
\mathrm{CA}=559(\mathrm{deg})
$$

$$
\mathrm{CA}=599(\mathrm{deg})
$$
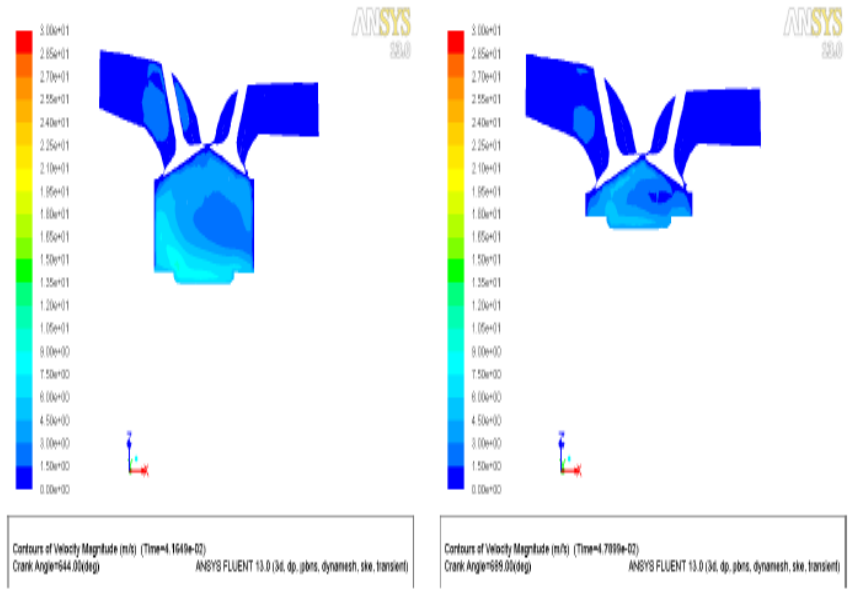

$$
\mathrm{CA}=644(\mathrm{deg})
$$

$\mathrm{CA}=689(\mathrm{deg})$

Fig 5 Velocity magnitude for CA 644 and 689

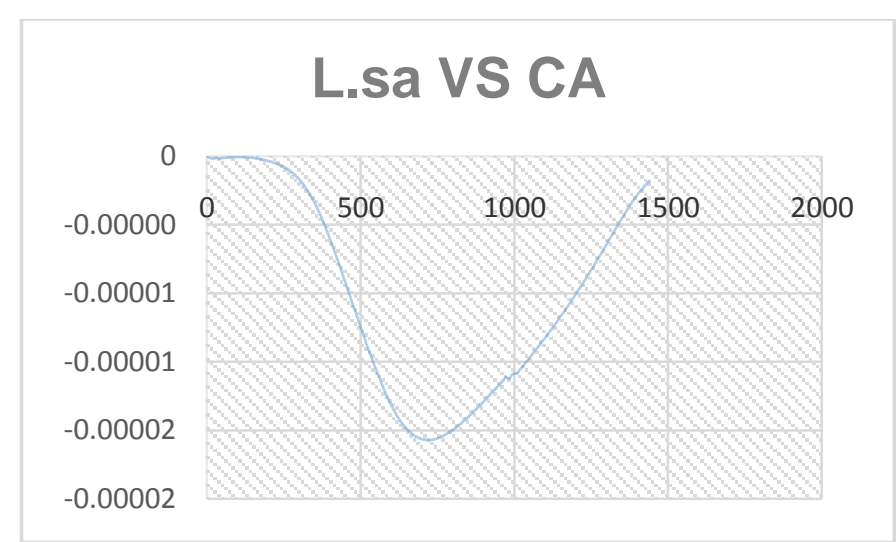

Fig 6 Swirl vs CA

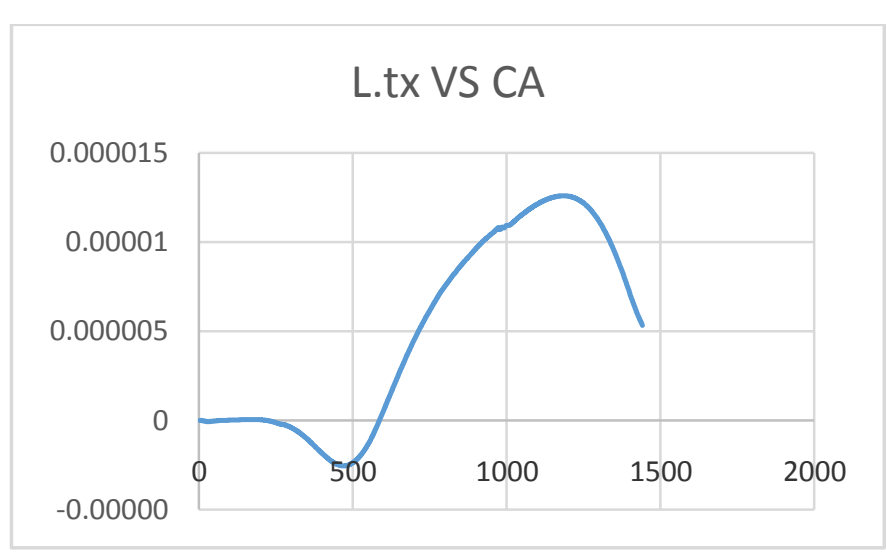

Fig 7 X-Tumble vs CA

Fig 4 Velocity magnitude for CA 559 and 599 


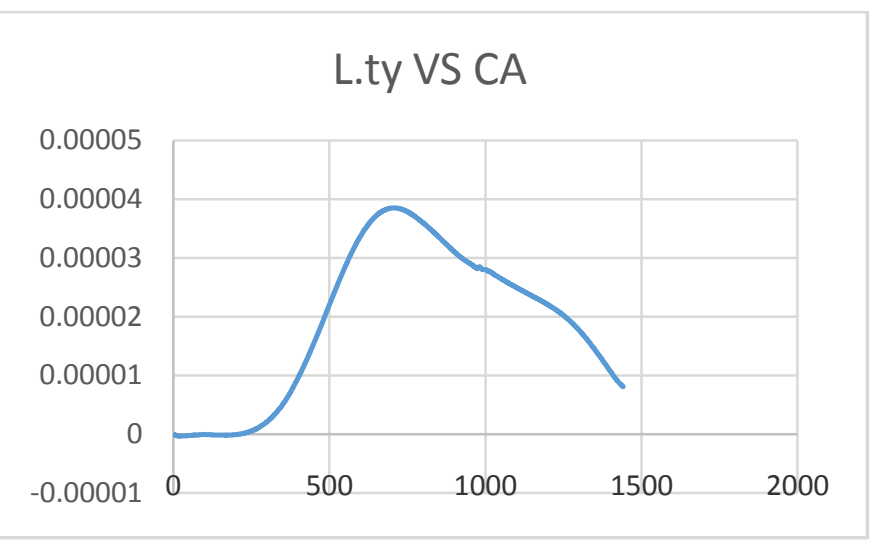

Fig 8 Y-Tumble vs CA

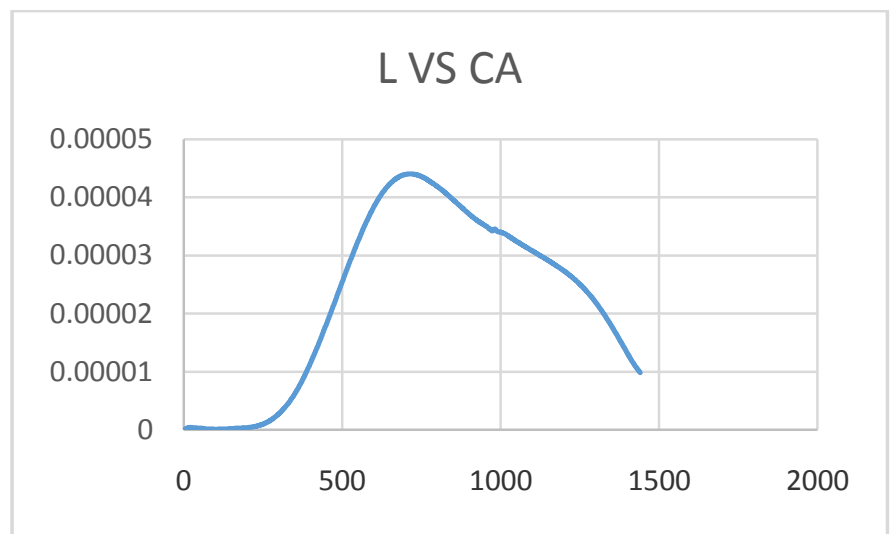

Fig 9 Angular momentum vector of fluid vs CA

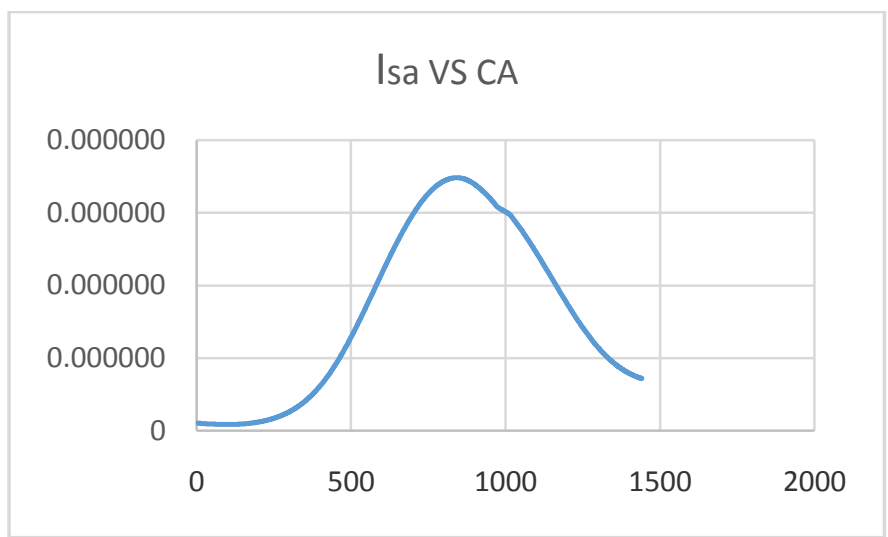

Fig 10 Moment of Inertia of fluid mass about swirl axis

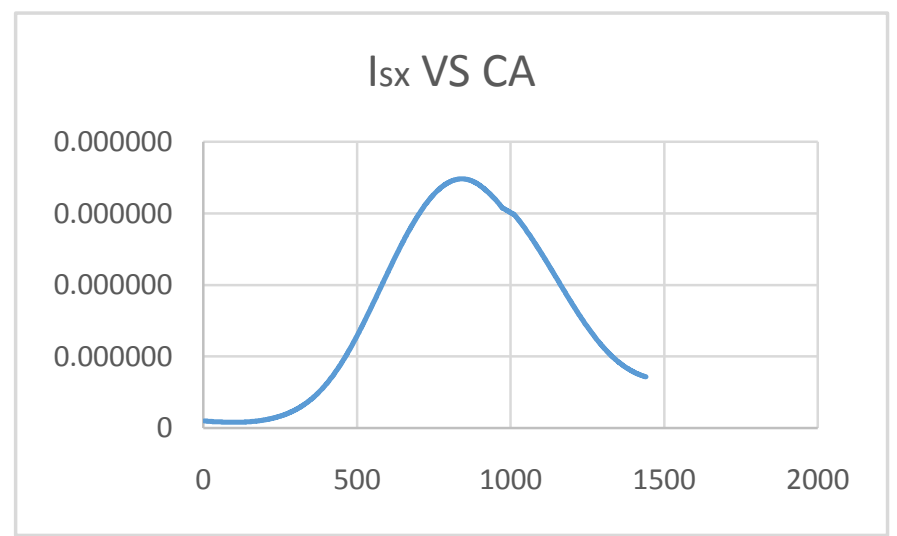

Fig 11 Moment of Inertia of the fluid mass about Tumble Xaxis

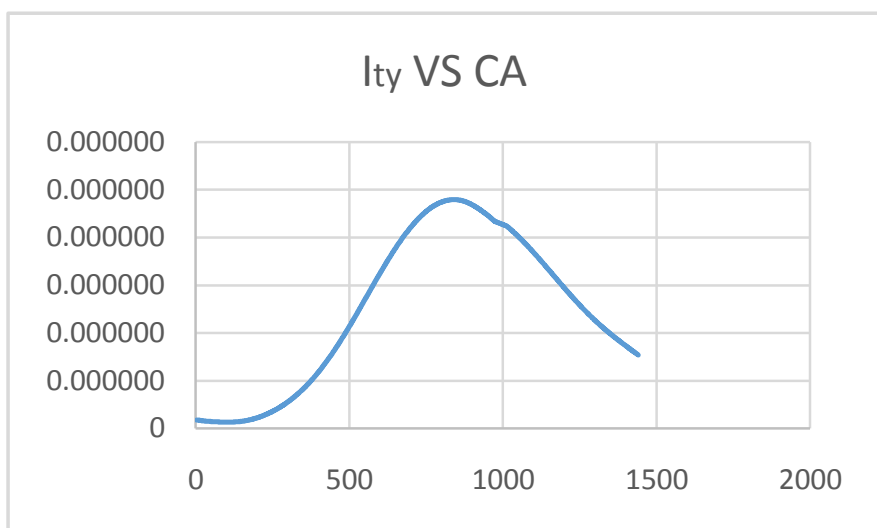

Fig 12 Moment of Inertia of the fluid mass about Tumble Yaxis

\section{CONCLUSIONS}

In this paper the In cylinder cold flow CFD simulation of four stroke petrol engine using hybrid approach of ANSYS fluent. The simulation is carried out using parameter and journal files which is a symmetry geometry. Dynamic motion was visualize and velocity magnitude is plotted for crank angle starting from 0 to 720 . The engine is simulated for half cylinder cycle. The In cylinder data file is write displaying swirl and tumble for zones of fluid-ch and fluidpiston-layer. The text file is written in working directory containing swirl, $\mathrm{x}$-tumble, y-tumble and moment of inertia as a function of CA.

\section{REFERENCES}

[1] T.P.Balaji Vignesh,C.Balamurugan, N.Vinayagam and T.Gavaskar "Experimental Analysis and Modelling of a Four Stroke Single Cylinder Di Diesel Engine Under Variable Compression Ratio" International Journal of Engineering Science and Technology (IJEST) ISSN : 0975-5462 Vol. 4 No.09 September 2012.

[2] Victor IORGA-SIMAN,Adrian CLENCI,Pierre PODEVIN,Alain DELACROIX and Ion TABACU "In Cylinder Flow Analysis of Different Valve Lift Using CFD" University of Pitesti,Scientific Bulletin, Automotive Series, vol. 21 (2).

[3] S. M. Jameel Basha,P. Issac Prasad and K. Rajagopal "Simulation of In - Cylinder Processes in a DI Diesel Engine with various Injection timings" ARPN Journal of Engineering and Applied Sciences,ISSN 1819-6608, VOL. 4, NO. 1, FEBRUARY 2009.

[4] Feilong Liu, Gehan A. J. Amaratunga and Nick Collings and Ahmed Soliman "An Experimental Study on Engine Dynamics Model Based In-Cylinder Pressure Estimation" SAE International 2012-010896 published 04/16/2012.

[5] Cao, Y., "Thermodynamic Cycles of Internal Combustion Engines for Increased Thermal Efficiency, Constant-Volume Combustion, Variable Compression Ratio, and Cold Start," SAE Technical Paper 2007-01-4115, 2007, doi:10.4271/2007-014115. 
[6] Eric Tribbett, Ed Froehlich, Lex Bayer "Effects of Ignition Timing, Equivalence Ratio and Copression Ratio on RDH Engine Performance.

[7] Sourabh Shrivastava, Padmesh Mandloi , Ajey Walavalkar, " Modeling IC Engine Thermal management using ANSYS' ANSYS Conference.

[8] Waidmann, W., Boemer, A. and Braun, M., "Adjustment and Verification of Model Parameters for Diesel Injection CFD Simulation", SAE Paper 2006-01-0241.

[9] Heywood, J., B., "Internal Combustion Engine Fundamentals", McGraw Hill International Editions (1988)

[10] 10) FLUENT 12, User's Guide, ANSYS Inc., Lebanon, NH, (2006).

[11] Ganeshan. V Advanced I.C. Engines 3rd ed, McGraw- Hill : 2008. 